\title{
Myelopathy and reactive microgliosis and astrogliosis in equine back pain
}

\begin{abstract}
Equine chronic back pain (CBP) has been linked to different pathologic processes, which directly or indirectly involve spinal structures. Thus, making diagnosis and management very challenging with most horses with the condition recommended for early retirement from athletic activity. This study described the spinal cord lesions and the development of reactive microgliosis and astrocytosis in the spinal cords of horse with CBP. Thoracolumbar spinal cord segments from three horses euthanized because of unresolved CBP were dissected and grossly and histopathologically examined. The expression of activated microglia and astrocytes were demonstrated immunohistochemically using polyclonal rabbit anti-Iba-1 and anti-glial fibrillary acidic protein antibodies, respectively. All horses had radiological evidence of varying degrees of kissing spine involving six to nine vertebrae with the majority of the lesions graded between 2 and 5. Grossly, there was myelomalacia with intramedullary hemorrhages. The gray matters of the spinal cords were characterized by hemorrhagic malacic lesions with medullary disintegration. Reactive microgliosis and astrocytosis were evident in the spinal dorsal horns. White matter lesions include axonal swollen and/or loss, satellitosis, and varying degrees of dilation of myelin sheaths with some containing macrophages. In conclusion, the presence of reactive microgliosis and astrogliosis in the spinal dorsal horn indicates that they are possible precipitating factors in the development of equine CBP.
\end{abstract}

Keyword: Spinal cord; Microgliosis; Astrocytosis; Chronic back pain; Horses 